\title{
MEASUREMENT OF GROUND WATER VELOCITY \\ USING RHODAMINE WT DYE NEAR \\ SHEFFIELD， ILLINOIS
}

By George Garklavs and L. G. Toler

U.S. GEOLOGICAL SURVEY

Open-File Report $84-856$

Prepared in cooperation with the

ILLINOIS DEPARTMENT OF NUCLEAR SAFETY

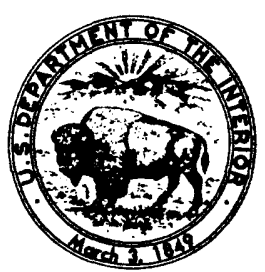

Urbana, Illinois 


\section{UNITED STATES DEPARTMENT OF THE INTERIOR \\ WILLIAM P. CLARK, Secretary}

GEOLOGICAL SURVEY

Dallas L. Peck, Director

For additional information

write to:

District Chief

U.S. Geological Survey Water Resources Division

4 th Floor

102 East Main street

Urbana, IL 61801
Copies of this report can be purchased from:

Open-File services section Western Distribution Branch U.S. Geological survey Box 25425, Federal Center Denver, CO 80225

[Telephone: (303) 234-5888] 


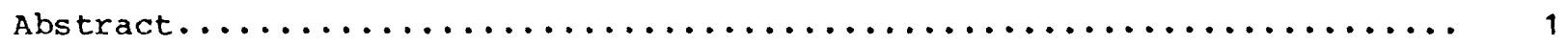

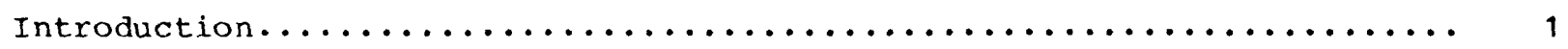

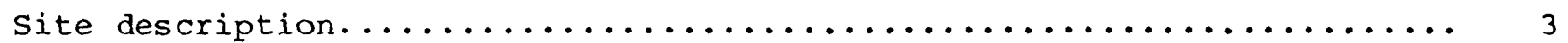

Methods................................................4

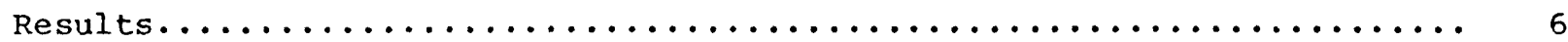

Ground-water flow velocity.............................

Factors affecting measured velocity......................11

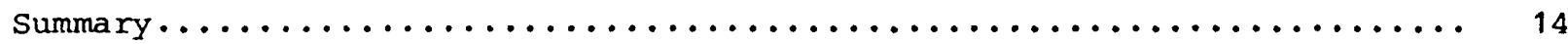

References cited.......................................

\section{ILLUSTRATIONS}

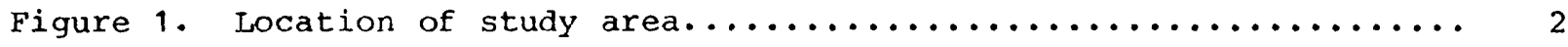

2. We11 locations and hydrogeologic section at measurement site..................................... 5

3. Sampling equipment for collecting dye samples............. 7

4. Concentration hydrographs of Rhodamine wT dye............ 12

TABLES

Table 1. Well depths and altitudes...........................4

2. Chemicals used in tracer test.........................6

3. Fluorometric analyses of Rhodamine wT dye................ 8

4. Chemical analyses of tracers......................... 11 


\section{CONVERSION FACTORS}

Multiply inch-pound unit

foot (ft)

acre

foot per day (ft/d)

foot per year (ft/yr)

galion (gal)

pint (pt)
By

0.3048

0.4047

0.3048

0.3048

3.785

0.4732
To obtain SI unit

meter (m)

hectare

meter per day (m/d)

meter per year ( $\mathrm{m} / \mathrm{yr})$

liter (L)

liter (L) 
MEASUREMENT OF GROUND-WATER VELOCITY USING RHODAMINE WT DYE

NEAR SHEFFIELD, ILLINOIS

by George Garklavs and I. G. Tolex

ABSTRACT

Ground-water flow velocity was estimated in a tract of land adjacent to a low-level radioactive-waste disposal site near sheffield, Illinois, by measuring the time of travel between two wells spaced 110 feet apart. Rhodamine WT dye was the principal tracer used in the test. The leading edge and peak concentrations of Rhodamine WT were well defined. A ground-water velocity of 6.9 feet per day $(2,500$ feet per year) was computed from the arrival time of the leading edge of the tracer cloud.

\section{INTRODUCTION}

Hydrogeologic studies (Foster, Erickson, and Healy, 1984; Foster, Garklavs, and Mackey, 1984) at a low-level radioactive-waste disposal site near Sheffield, Illinois (fig. 1), have revealed a pebbly-sand unit that is believed to convey most of the subsurface drainage from about two-thirds of the 20-acre site. Offsite to the northeast, in the sand unit, most of the ground-water flow seemingly is through a sand-filled channel in an underlying clayey-silt unit.

Radiometric analyses have defined a plume of tritium in ground water extending eastward fxom the site toward a surface-mine lake located about 800 feet from the site boundary. The tritium plume is coincident with the sandfilled channel and is about 30 to 50 feet wide.

Foster, Exickson, and Healy (1984, p. 23) calculated that water velocities in the sand unit might range fxom 0.67 to 1,600 feet per year, with the higher velocities being offsite to the east. Confirmation of the potentially higher rate of water movement was needed to better define the hydrogeology and to provide information for site planners to make knowledgeable decisions with regard to potential radionuclide movement.

The purpose of this report is to give the results of using RWT (Rhodamine WT) dye to measure ground-water velocity in the sand unit. The measurements are limited to one small part of the sand unit. The field procedures are described, and the results are presented as tables of concentrations and concentration hydrographs showing time of arrival of the RWT at observation wells. 

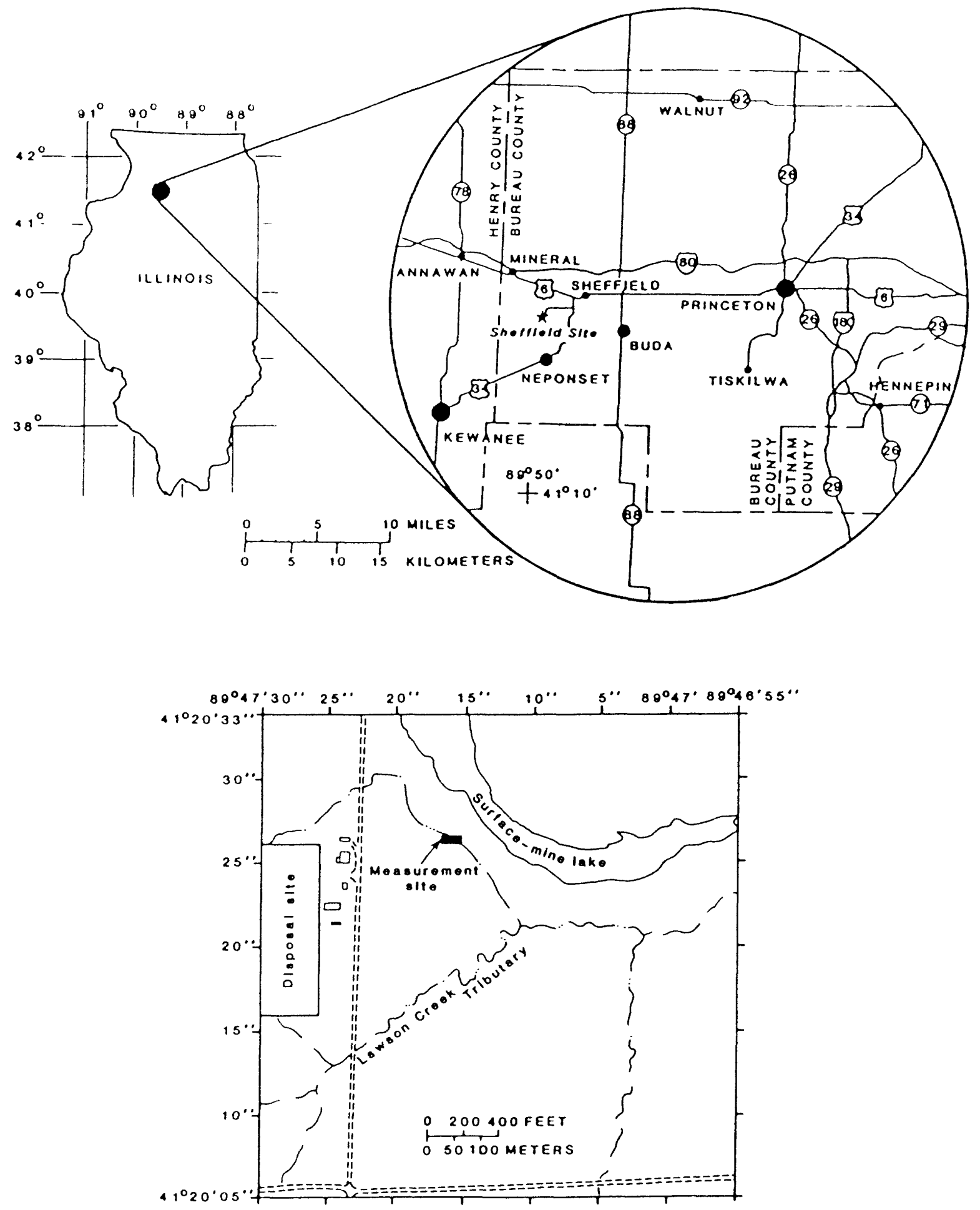

Figure 1.--Location of the study area. 
The study was conducted in cooperation with the Illinois Department of Nuclear Safety. Dr. Terry Lash was particularly helpful in encouraging and supporting this effort. Personnel of US Ecology, operators of the disposal site, assisted in the field efforts and collected most of the samples. Andy Armbrust, of US Ecology was especially helpful in collecting samples, making water-level measurements, and coordinating field efforts. Dr. Glenn Thompson, Tracer Research Corporation, Tucson, Arizona, provided all tracers and tracer analyses exclusive of Rhodamine WT and chloride.

\section{SITE DESCRIPTION}

The study area includes the Sheffield low-level waste-disposal site and adjoining lands (fig. 1)--an area of rolling terrain. The measurement site is in a tract of land adjacent to, and east of, the disposal site. The tract of land slopes gently to the east. Vegetation is sparse and some gullying has occurred throughout the tract.

Quaternary unconsolidated materials overlie Pennsylvanian bedrock in the study area. The quaternary materials range in thickness from 10 to 80 feet and consist of till, glacial outwash and lacustrine deposits, and loess. All hydrogeologic descriptions of the study area are taken from reports by Foster, Erickson, and Healy (1984) and from Foster, Garklavs, and Mackey (1984).

The principal water-bearing unit in the study area is a pebbly-sand unit of the Toulon Member of the Glasford Formation. The pebbly-sand unit ranges from 1 to 35 feet in thickness where present, and grades from a moderately sorted, pebbly, silty sand in the disposal site to a well sorted, pebbly sand throughout much of the study area. The unit underlies about two-thirds of the disposal site and much of the adjoining area to the northeast. Much of the underground drainage from the disposal site is believed to be through this pebbly-sand unit.

Northeastward from the disposal site, the pebbly-sand unit fills a shallow, channel-like depression in the underlying Hulick Till Member. This gravelly, pebbly, sand-filled channel provides a conduit for ground-water flow from the northeast corner of the disposal site east-northeast to a surfacemine lake.

Radiometric analyses for tritium have defined a plume of tritium in ground water extending northeastward from the disposal site that is roughly coincident with the sand-filled channel. The tritium is apparently limited to an area about 30 to 50 feet wide which is only slightly wider than the sandfilled channel.

Ground-water flow in the study area is generally toward the northeast. Much of the flow is through the pebbly-sand unit. The principal path, off site, seems to be through the channel-fill part of the unit. 


\section{METHODS}

The measurement site (fig. 1) was selected to measure ground-water velocity along the sand channel in order to better define time of travel to the surface-mine lake. Well 601 (fig. 2) was used for injecting the tracers. Before the test, a 5-foot length of pipe extended below the well screen and acted as a silt trap. This extension was packed off to avoid having tracers trapped in the extension and released to the aquifer after the original injection. Well 601 is 27 feet deep and is screened in the bottom 10 feet of the sand unit. During the test, the water level in the well was below the top of the screen.

Five observation wells ( $f$ ig. 2) were installed downgradient of well 601 for the purpose of collecting samples for field and laboratory analyses of the tracers. The locations of the observation wells were selected to be downgradient of the injection well and within the channel fill and the tritium plume. Wells $T-1$ to $T-5$ are $12,12,10,10$, and 9 feet deep, respectively. A 2-foot screen at the bottom of each observation well is in the pebbly sand except well $\mathrm{T}-4$ which has only 1 foot of screen open to the sand (table 1 ).

Table $1 .-$-Well depths and altitudes

\begin{tabular}{|c|c|c|c|c|c|}
\hline \multirow[b]{2}{*}{$\begin{array}{l}\text { Well } \\
\text { No. }\end{array}$} & \multirow{2}{*}{$\begin{array}{l}\text { Depth } \\
\text { of } \\
\text { well } \\
\text { (feet) }\end{array}$} & \multicolumn{4}{|c|}{ Altitude, in feet above sea level } \\
\hline & & $\begin{array}{c}\text { Land } \\
\text { surface }\end{array}$ & $\begin{array}{l}\text { Screened } \\
\text { interval }\end{array}$ & $\begin{array}{c}\text { Sand } \\
\text { interval }\end{array}$ & $\begin{array}{c}\text { Water } \\
\text { surface }\end{array}$ \\
\hline 601 & 27 & 729.8 & $702 \cdot 8-712 \cdot 8$ & $702 \cdot 8-725 \cdot 8$ & 710.1 \\
\hline$T-1$ & 12 & 715.7 & $704.2-706.2$ & $702.2-713.7$ & 709.1 \\
\hline $\mathrm{T}-2$ & 12 & $715 \cdot 3$ & $703.8-705.8$ & $704 \cdot 0-710 \cdot 8$ & 708.8 \\
\hline $\mathrm{T}-3$ & 10 & 715.1 & $705.6-707.6$ & $702 \cdot 8-713 \cdot 1$ & 708.9 \\
\hline $\mathrm{T}-4$ & 10 & 714.5 & $705.0-707.0$ & $706.0-712.5$ & 708.7 \\
\hline$T-5$ & 9 & 714.6 & $705.9-707.9$ & $704.3-711.8$ & 708.6 \\
\hline
\end{tabular}

1 Water levels measured on January $3,1984$.

Radiometric analyses for tritium in water from wells $T-1$ through $T-5$ were used to determine if these wells were in the plume of tritium. Analyses of tritium in these wells showed concentrations of the same magnitude as elsewhere along the plume, indicating that the observation wells were within the tritium plume.

Five tracers (table 2) were injected into well 601. All tracers were combined into about 1.25 gallons of well-mixed solution. The solution was poured down the injection well from narrow-mouth glass containers. The containers were rinsed with an additional 1.25 gallons of distilled water and the rinse water was also poured into the injection well to cleanse the well wall of the tracer solution. The solution was again mixed within the well by raising and lowering a one-half pint capacity plastic (PVC) bailer for about 5 minutes. No effort was made to force the solution from the well into the sand unit. 

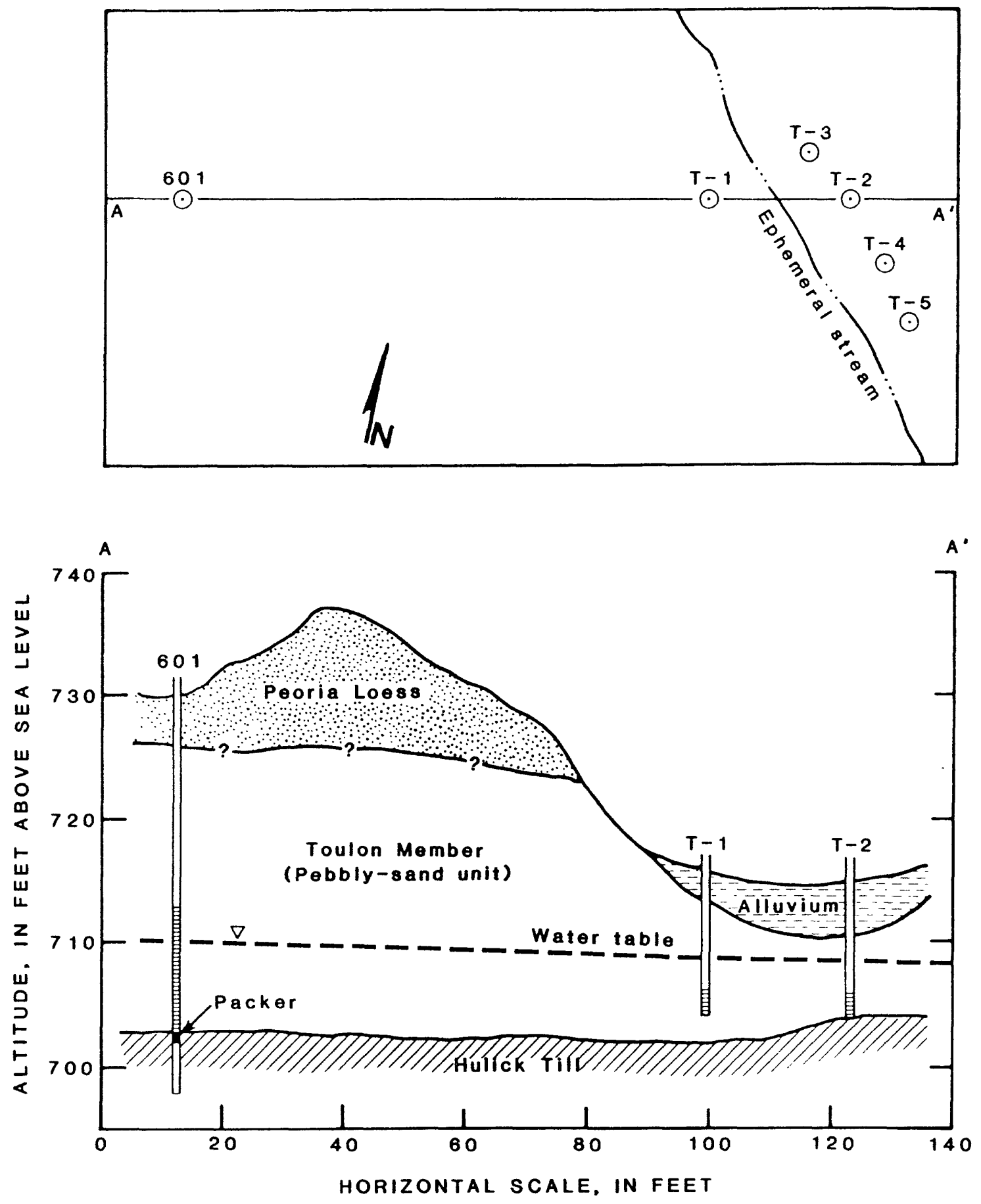

Figure 2.--Well locations and hydrogeologic section at measurement site. 
Table 2.--Chemicals used in tracer test

\begin{tabular}{lcc}
\hline \multicolumn{1}{c}{ Name } & $\begin{array}{c}\text { Abbrevi- } \\
\text { ation }\end{array}$ & Amount \\
\hline Rhodamine WT & RWT & 400 grams of 20 percent solution \\
Trifluoroacetate & TFA & 500 grams \\
m-Trifluoromethylbenzoate & m-TFMB & 400 grams \\
Pyridone & PYD & 500 grams, 80 percent pure \\
Pentafluoropropionate & PFP & 500 grams \\
\hline
\end{tabular}

Concurrently with injecting tracers into well 601, about 1 gallon of a solution having an approximate concentration of 1,000 milligrams per liter calcium chloride was injected into well $\mathrm{T}-1$. Analyses of chloride in samples from wells $\mathrm{T}-2$ and $\mathrm{T}-4$ were to be used to estimate velocities in the short reach from well $\mathrm{T}-1$ in order to compute the time of arrival at well $\mathrm{T}-1$ of the other tracers injected in well 601. The frequency of sampling was to be increased when the tracers injected in well 601 were near well $T-1$, based on the computed velocity between wells $\mathrm{T}-1$ and $\mathrm{T}-2$ or $\mathrm{T}-4$.

The five observation wells, $T-1$ through $\mathrm{T}-5$, were equipped with sampling tubes that remained in place during the tracer test. Samples of water were collected using these tubes and the sampling apparatus shown in figure 3 . The hand-operated vacuum pump was used to pump about one-quarter pint of water with which to flush the sampling tube and rinse the vacuum flask prior to collecting each sample. After flushing the tube and rinsing the flask, a sample volume of about one-half pint was collected. Each of the observation wells was assigned a separate flask to avoid the possibility of tracer contamination between wells.

Analyses of chloride were made at the site using a field test kit. Rhodamine WT analyses were performed by personnel of the U.S. Geological Survey in Urbana, Illinois. Analyses of other tracers were performed by Tracer Research Corporation, Tucson, Arizona.

\section{RESULTS}

\section{Ground-Water Flow Velocity}

The tracers were injected into wells 601 and $T-1$ on January 24, 1984, at 10:30 a.m. Wells $T-1, T-2$, and $T-4$ were sampled daily at 8:00 a.m. Although no chloride was detected in wells $\mathrm{T}-2$ or $\mathrm{T}-4$, a pink color was visible on February 15, in samples from wells $\mathrm{T}-1$ and $\mathrm{T}-2$. Sampling frequency in all observation wells was increased to three times per day; at midnight, 8:00 a.m. and 4:00 p.m. This sampling schedule was continued until March 24, at which time the sampling frequency was reduced to once daily. Sampling was discontinued on May 14, 1984. The analyses for RWT are given in table 3. Only selected samples were analyzed to determine the arrival time of the tracers 


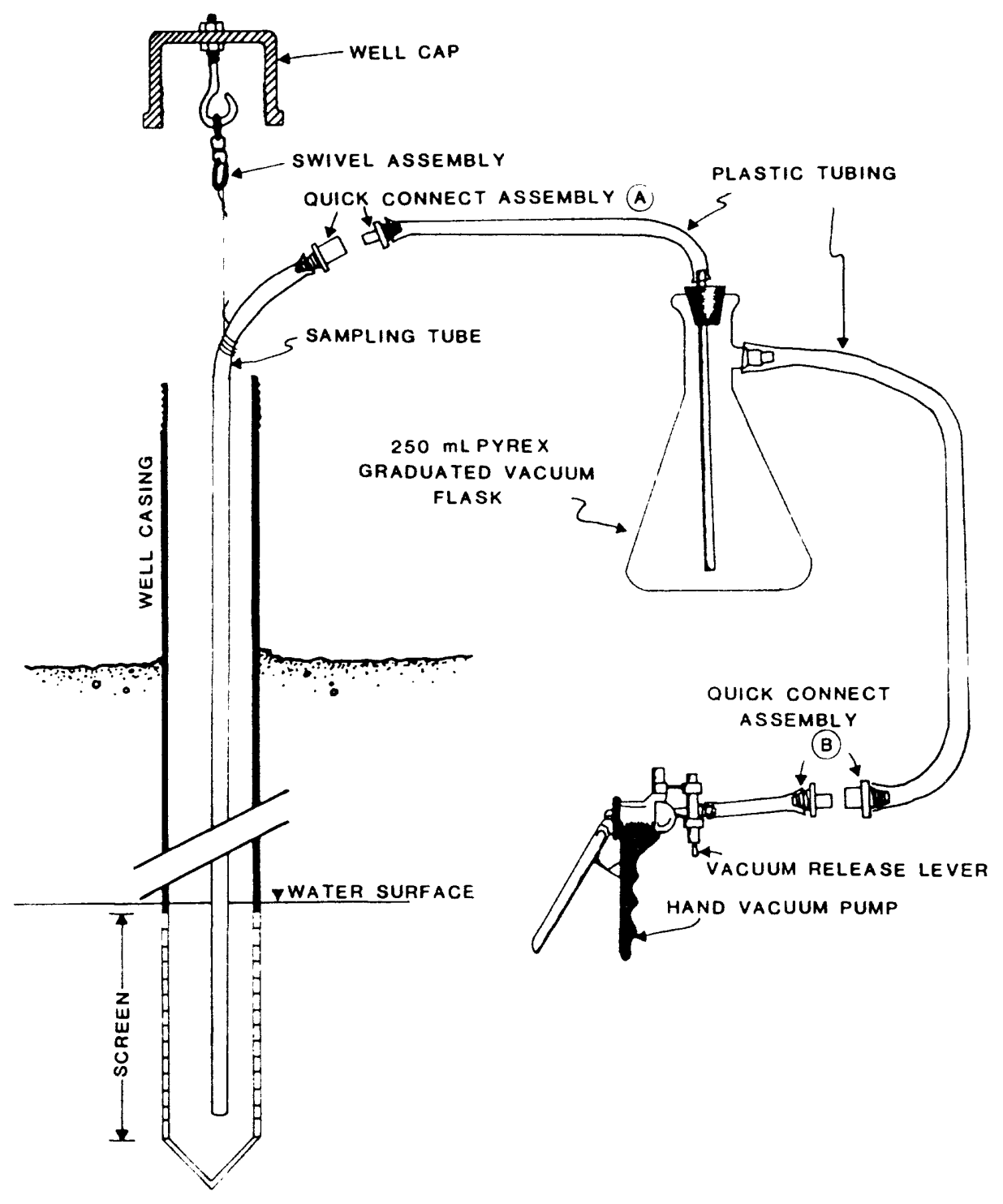

Figure 3.--Sampling equipment for collecting dye samples. 
Table 3.--Fluorometric analyses of Rhodamine WT dye

[Concentrations in micrograms per liter;

* after time indicates time estimated]

\begin{tabular}{|c|c|c|c|c|c|c|c|}
\hline $\begin{array}{l}\text { Well } \\
\text { No. }\end{array}$ & Date & Time & $\begin{array}{l}\text { Concen- } \\
\text { tration }\end{array}$ & $\begin{array}{l}\text { Well } \\
\text { No. }\end{array}$ & Date & Time & $\begin{array}{l}\text { Concen- } \\
\text { tration }\end{array}$ \\
\hline$T-1$ & $01-23-84$ & $0800^{\star}$ & 0.0 & $T-1$ & $03-30-84$ & 1200 & 144 \\
\hline$T-1$ & $02-01-84$ & $0800^{*}$ & .0 & $T-1$ & $03-30-84$ & 1600 & 134 \\
\hline$T-1$ & $02-03-84$ & 0800 * & .0 & $T-1$ & $03-31-84$ & 0800 & 128 \\
\hline$T-1$ & $02-05-84$ & $0800^{*}$ & .0 & $T-1$ & $03-31-84$ & 1200 & 131 \\
\hline$T-1$ & $02-06-84$ & $0800^{\star}$ & .0 & $T-1$ & $03-31-84$ & 1600 & 128 \\
\hline$T-1$ & $02-07-84$ & $0800^{*}$ & 2.1 & $T-1$ & $04-01-84$ & 0800 & 135 \\
\hline$T-1$ & $02-08-84$ & $0800^{\star}$ & 8.1 & $T-1$ & $04-01-84$ & 1200 & 129 \\
\hline$T-1$ & $02-09-84$ & $0800^{\star}$ & 8.2 & $T-1$ & $04-01-84$ & 1600 & 134 \\
\hline$T-1$ & $02-10-84$ & $0800 *$ & 65 & $T-1$ & $04-02-84$ & 0800 & 127 \\
\hline$T-1$ & $02-11-84$ & $0800 *$ & 121 & $T-1$ & $04-02-84$ & 1200 & 125 \\
\hline$T-1$ & $02-13-84$ & 0800 * & 276 & $T-1$ & $04-02-84$ & 1600 & 127 \\
\hline $\mathrm{T}-1$ & $02-15-84$ & $0800 *$ & 214 & $T-1$ & $04-03-84$ & 0800 & 128 \\
\hline$T-1$ & $02-17-84$ & 0800 & 383 & $T-1$ & $04-03-84$ & 1200 & 127 \\
\hline$T-1$ & $02-19-84$ & 0800 & 394 & $T-1$ & $04-03-84$ & 1600 & 119 \\
\hline$T-1$ & $02-21-84$ & 0800 & 464 & $T-1$ & $04-04-84$ & 0800 & 122 \\
\hline$T-1$ & $02-23-84$ & 0800 & 455 & $T-1$ & $04-05-84$ & 0800 & 124 \\
\hline$T-1$ & $02-25-84$ & 0800 & 446 & $T-1$ & $04-06-84$ & 0800 & 131 \\
\hline$T-1$ & $02-27-84$ & 0800 & 383 & $T-1$ & $04-07-84$ & 0800 & 102 \\
\hline$T-1$ & $02-29-84$ & 0800 & 382 & $T-1$ & $04-08-84$ & 0800 & 128 \\
\hline$T-1$ & $03-04-84$ & 0800 & 378 & $T-1$ & $04-09-84$ & 0800 & 122 \\
\hline$T-1$ & $03-06-84$ & 0800 & 346 & $T-1$ & $04-10-84$ & 0800 & 162 \\
\hline$T-1$ & $03-08-84$ & 0800 & 348 & $T-1$ & $04-11-84$ & 0800 & 165 \\
\hline$T-1$ & $03-10-84$ & 0800 & 232 & $T-1$ & $04-12-84$ & 0800 & 168 \\
\hline$T-1$ & $03-12-84$ & 0800 & 303 & $T-1$ & $04-13-84$ & 0800 & 179 \\
\hline$T-1$ & $03-14-84$ & 0800 & 260 & $T-1$ & $04-14-84$ & 0800 & 128 \\
\hline$T-1$ & $03-16-84$ & 0800 & 180 & $T-1$ & $04-15-84$ & 0800 & 129 \\
\hline$T-1$ & $03-18-84$ & 0800 & 183 & $T-1$ & $04-16-84$ & 0800 & 134 \\
\hline $\mathrm{T}-1$ & $03-20-84$ & 0800 & 251 & $T-1$ & $04-17-84$ & 0800 & 168 \\
\hline$T-1$ & $03-22-84$ & 2400 & 364 & $T-1$ & $04-18-84$ & 0800 & 125 \\
\hline$T-1$ & $03-24-84$ & 0800 & 178 & $T-1$ & $04-19-84$ & $0800^{*}$ & 151 \\
\hline$T-1$ & $03-25-84$ & 1200 & 131 & $T-1$ & $04-20-84$ & 0800 & 159 \\
\hline$T-1$ & $03-26-84$ & 0800 & 137 & $T-1$ & $04-21-84$ & $0800^{\star}$ & 134 \\
\hline$T-1$ & $03-26-84$ & 0800 & 137 & $T-1$ & $04-22-84$ & 0800 & 117 \\
\hline$T-1$ & $03-26-84$ & 1200 & 134 & $T-1$ & $04-23-84$ & 0800 & 171 \\
\hline$T-1$ & $03-26-84$ & 1200 & 137 & $T-1$ & $04-24-84$ & 0800 & 141 \\
\hline$T-1$ & $03-26-84$ & 1600 & 128 & $T-1$ & $04-25-84$ & 0800 & 179 \\
\hline$T-1$ & $03-27-84$ & 0800 & 137 & $T-1$ & $04-26-84$ & 0800 & 199 \\
\hline$T-1$ & $03-27-84$ & 1200 & 134 & $T-1$ & $04-27-84$ & 0800 & 191 \\
\hline$T-1$ & $03-27-84$ & 1600 & 135 & $T-1$ & $05-07-84$ & 0800 & 201 \\
\hline$T-1$ & $03-28-84$ & 0800 & 139 & $T-1$ & $05-08-84$ & 0800 & 202 \\
\hline$T-1$ & $03-28-84$ & 0800 & 137 & $T-1$ & $05-09-84$ & 0800 & 217 \\
\hline$T-1$ & $03-28-84$ & 1600 & 98 & $T-1$ & $05-10-84$ & 0800 & 205 \\
\hline$T-1$ & $03-29-84$ & 0800 & 124 & $T-1$ & $05-11-84$ & 0800 & 75 \\
\hline$T-1$ & $03-29-84$ & 1200 & 139 & $T-1$ & $05-11-84$ & $1200^{*}$ & 194 \\
\hline$T-1$ & $03-29-84$ & 1600 & 124 & $T-1$ & $05-12-84$ & 0800 & 197 \\
\hline$T-1$ & $03-30-84$ & 0800 & 139 & $T-1$ & $05-13-84$ & 0800 & 199 \\
\hline
\end{tabular}


Table 3.--Fluorometric analyses of Rhodamine WT dye--Continued

\begin{tabular}{|c|c|c|c|c|c|c|c|}
\hline $\begin{array}{l}\text { Well } \\
\text { No. }\end{array}$ & Date & Time & $\begin{array}{l}\text { Concen- } \\
\text { tration }\end{array}$ & $\begin{array}{l}\text { Well } \\
\text { No. }\end{array}$ & Date & Time & $\begin{array}{l}\text { Concen- } \\
\text { tration }\end{array}$ \\
\hline $\mathrm{T}-2$ & $02-01-84$ & $0800^{\star}$ & 0.0 & $T-2$ & $03-30-84$ & 1600 & 2.5 \\
\hline $\mathrm{T}-2$ & $02-03-84$ & $0800 \star$ & .0 & $T-2$ & $03-31-84$ & 0800 & 2.6 \\
\hline $\mathrm{T}-2$ & $02-05-84$ & 0800 * & .0 & $T-2$ & $03-31-84$ & 1200 & 2.5 \\
\hline$T-2$ & $02-06-84$ & $0800 *$ & .0 & $T-2$ & $03-31-84$ & 1600 & 2.6 \\
\hline $\mathrm{T}-2$ & $02-07-84$ & $0800^{*}$ & .0 & $T-2$ & $04-01-84$ & 0800 & 2.4 \\
\hline$T-2$ & $02-08-84$ & 0800 * & .0 & $T-2$ & $04-01-84$ & 1200 & 2.2 \\
\hline $\mathrm{T}-2$ & $02-09-84$ & $0800^{\star}$ & .5 & $T-2$ & $04-01-84$ & 1600 & 2.4 \\
\hline $\mathrm{T}-2$ & $02-10-84$ & $0800^{*}$ & 3.5 & $T-2$ & $04-02-84$ & 0800 & 2.4 \\
\hline$T-2$ & $02-11-84$ & $0800 *$ & 7.7 & $T-2$ & $04-02-84$ & 1200 & 2.3 \\
\hline $\mathrm{T}-2$ & $02-13-84$ & 0800 * & 84 & $T-2$ & $04-02-84$ & 1600 & 2.3 \\
\hline $\mathrm{T}-2$ & $02-15-84$ & 0800 * & 64 & $\mathrm{~T}-2$ & $04-03-84$ & 0800 & 2.3 \\
\hline $\mathrm{T}-2$ & $02-17-84$ & 0800 & 28 & $\mathrm{~T}-2$ & $04-03-84$ & 1200 & 2.6 \\
\hline $\mathrm{T}-2$ & $02-19-84$ & 0800 & 19 & $T-2$ & $04-03-84$ & 1600 & 2.4 \\
\hline $\mathrm{T}-2$ & $02-21-84$ & 0800 & 21 & $T-2$ & $04-04-84$ & 0800 & 2.5 \\
\hline $\mathrm{T}-2$ & $02-23-84$ & 0800 & 18 & $T-2$ & $04-05-84$ & 0800 & 2.9 \\
\hline $\mathrm{T}-2$ & $02-25-84$ & 0800 & 16 & $T-2$ & $04-06-84$ & 0800 & 1.6 \\
\hline $\mathrm{T}-2$ & $02-27-84$ & 0800 & 13 & $\mathrm{~T}-2$ & $04-07-84$ & 0800 & 2.5 \\
\hline $\mathrm{T}-2$ & $02-29-84$ & 0800 & 12 & $T-2$ & $04-08-84$ & 0800 & 1.7 \\
\hline $\mathrm{T}-2$ & $03-04-84$ & 0800 & 16 & $\mathrm{~T}-2$ & $04-09-84$ & 0800 & 1.7 \\
\hline $\mathrm{T}-2$ & $03-06-84$ & 0800 & 17 & $\mathrm{~T}-2$ & $04-10-84$ & 0800 & 1.8 \\
\hline $\mathrm{T}-2$ & $03-08-84$ & 0800 & 17 & $\mathrm{~T}-2$ & $04-11-84$ & 0800 & 2.8 \\
\hline$T-2$ & $03-10-84$ & 0800 & 17 & $\mathrm{~T}-2$ & $04-12-84$ & 0800 & 1.9 \\
\hline$T-2$ & $03-12-84$ & 0800 & 16 & $T-2$ & $04-13-84$ & 0800 & 3.2 \\
\hline$T-2$ & $03-14-84$ & 0800 & 16 & $r-2$ & $04-14-84$ & 0800 & 3.4 \\
\hline $\mathrm{T}-2$ & $03-16-84$ & 0800 & 15 & $T-2$ & $04-14-84$ & 0800 & 2.9 \\
\hline $\mathrm{T}-2$ & $03-18-84$ & 0800 & 15 & $T-2$ & $04-15-84$ & 0800 & 2.6 \\
\hline $\mathrm{T}-2$ & $03-20-84$ & 0800 & 11 & $T-2$ & $04-16-84$ & 0800 & 3.7 \\
\hline $\mathrm{T}-2$ & $03-22-84$ & 0800 & 9.1 & $T-2$ & $04-17-84$ & 0800 & 3.7 \\
\hline $\mathrm{T}-2$ & $03-24-84$ & 0800 & 8.0 & $T-2$ & $04-18-84$ & 0800 & 3.5 \\
\hline $\mathrm{T}-2$ & $03-25-84$ & 1200 & 5.9 & $T-2$ & $04-19-84$ & $0800^{*}$ & 3.9 \\
\hline $\mathrm{T}-2$ & $03-25-84$ & 1200 & 6.2 & $T-2$ & $04-20-84$ & 0800 & 4.2 \\
\hline$T-2$ & $03-25-84$ & 1600 & 5.0 & $T-2$ & $04-21-84$ & 0800 * & 3.3 \\
\hline $\mathrm{T}-2$ & $03-26-84$ & 0800 & 5.9 & $T-2$ & $04-22-84$ & 0800 & 2.5 \\
\hline$T-2$ & $03-26-84$ & 1200 & 4.7 & $T-2$ & $04-23-84$ & 0800 & 3.4 \\
\hline$T-2$ & $03-26-84$ & 1600 & 5.0 & $T-2$ & $04-24-84$ & 0800 & 3.2 \\
\hline$T-2$ & $03-27-84$ & $0800^{\star}$ & 3.4 & $T-2$ & $04-25-84$ & 0800 & 2.6 \\
\hline $\mathrm{T}-2$ & $03-27-84$ & $0800 *$ & 4.0 & $T-2$ & $04-26-84$ & 0800 & 2.1 \\
\hline$T-2$ & $03-27-84$ & 1200 & 4.7 & $T-2$ & $05-07-84$ & 0800 & .54 \\
\hline$T-2$ & $03-27-84$ & 1600 & 3.9 & $T-2$ & $05-08-84$ & 0800 & .54 \\
\hline$T-2$ & $03-28-84$ & 1200 & 2.2 & $T-2$ & $05-09-84$ & 0800 & .50 \\
\hline$T-2$ & $03-28-84$ & 1600 & 2.3 & $T-2$ & $05-10-84$ & 0800 & .40 \\
\hline$T-2$ & $03-29-84$ & 0800 & 3.2 & $T-2$ & $05-11-84$ & 0800 & 7.9 \\
\hline $\mathrm{T}-2$ & $03-29-84$ & 1200 & 2.9 & $T-2$ & $05-12-84$ & 0800 & 3.4 \\
\hline$T-2$ & $03-29-84$ & 1600 & 3.1 & $T-2$ & $05-13-84$ & 0800 & 3.4 \\
\hline$T-2$ & $03-30-84$ & 0800 & 2.9 & $\mathrm{~T}-2$ & $05-14-84$ & $0800 *$ & 3.4 \\
\hline $\mathrm{T}-2$ & $03-30-84$ & 1200 & 2.6 & & & & \\
\hline
\end{tabular}


Table 3.--Fluorometric analyses of Rhodamine WT dye--Continued

\begin{tabular}{|c|c|c|c|c|c|c|c|}
\hline $\begin{array}{l}\text { We } 11 \\
\text { No. }\end{array}$ & Date & Time & $\begin{array}{l}\text { Concen- } \\
\text { tration }\end{array}$ & $\begin{array}{l}\text { Well } \\
\text { No. }\end{array}$ & Date & Time & $\begin{array}{l}\text { Concen- } \\
\text { tration }\end{array}$ \\
\hline$T-3$ & $02-16-84$ & $0800^{\star}$ & 0.0 & $\mathrm{~T}-3$ & $04-03-84$ & 0800 & 0.0 \\
\hline $\mathrm{T}-3$ & $02-19-84$ & 0800 & .0 & $\mathrm{~T}-3$ & $04-03-84$ & 1200 & .0 \\
\hline $\mathrm{T}-3$ & $02-20-84$ & 0800 & .0 & $T-3$ & $04-04-84$ & 0800 & .0 \\
\hline$T-3$ & $02-24-84$ & 0800 & .0 & $T-3$ & $04-06-84$ & 0800 & .0 \\
\hline$T-3$ & $02-28-84$ & 0800 & .0 & $T-3$ & $04-07-84$ & 0800 & .0 \\
\hline $\mathrm{T}-3$ & $03-10-84$ & 0800 & .0 & $\mathrm{~T}-3$ & $04-10-84$ & 0800 & .0 \\
\hline$T-3$ & $03-23-84$ & 0800 & .0 & $T-3$ & $04-11-84$ & 0800 & .0 \\
\hline$T-3$ & $03-25-84$ & 1200 & .0 & $T-3$ & $04-12-84$ & 0800 & .0 \\
\hline $\mathrm{T}-3$ & $03-26-84$ & 0800 & .0 & $T-3$ & $04-13-84$ & 0800 & .0 \\
\hline$T-3$ & $03-26-84$ & 1200 & .0 & $T-3$ & $04-14-84$ & 0800 & .0 \\
\hline $\mathrm{T}-3$ & $03-27-84$ & 0800 & .0 & $T-3$ & $04-16-84$ & 0800 & .0 \\
\hline$T-3$ & $03-27-84$ & 1600 & .0 & $\mathrm{~T}-3$ & $04-20-84$ & 0800 & .0 \\
\hline$T-3$ & $03-28-84$ & 1200 & .0 & $T-3$ & $04-21-84$ & $0800 *$ & .0 \\
\hline$T-3$ & $03-29-84$ & 1200 & .0 & $T-3$ & $04-25-84$ & 0800 & .0 \\
\hline$T-3$ & $03-29-84$ & 1600 & .0 & $\mathrm{~T}-3$ & $04-26-84$ & 0800 & .0 \\
\hline$T-3$ & $03-30-84$ & 1200 & .0 & $T-3$ & $05-09-84$ & 0800 & .0 \\
\hline$T-3$ & $03-31-84$ & 1600 & .0 & $\mathrm{~T}-3$ & $05-09-84$ & 0800 & .0 \\
\hline$T-3$ & $04-01-84$ & 0800 & .0 & $\mathrm{~T}-3$ & $05-11-84$ & 0800 & .0 \\
\hline$T-3$ & $04-02-84$ & 1600 & .0 & $\mathrm{~T}-3$ & $05-12-84$ & 0800 & .0 \\
\hline $\mathrm{T}-4$ & $01-26-84$ & $0800 *$ & 0.0 & $T-4$ & $03-31-84$ & 1200 & 0.0 \\
\hline$T-4$ & $02-03-84$ & $0800 *$ & .0 & $T-4$ & $03-31-84$ & 1600 & .0 \\
\hline$T-4$ & $02-06-84$ & $0800 *$ & .0 & $\mathrm{~T}-4$ & $04-02-84$ & 1200 & .0 \\
\hline$T-4$ & $02-08-84$ & $0800 *$ & .0 & $T-4$ & $04-02-84$ & 1600 & .0 \\
\hline$T-4$ & $02-11-84$ & $0800 *$ & .0 & $T-4$ & $04-03-84$ & 1600 & .0 \\
\hline $\mathrm{T}-4$ & $02-15-84$ & $0800 *$ & .0 & $\mathrm{~T}-4$ & $04-04-84$ & 0800 & .0 \\
\hline $\mathrm{T}-4$ & $02-28-84$ & 0800 & .0 & $T-4$ & $04-07-84$ & 0800 & .0 \\
\hline$T-4$ & $03-23-84$ & 0800 & .0 & $\mathrm{~T}-4$ & $04-09-84$ & 0800 & .0 \\
\hline $\mathrm{T}-4$ & $03-25-84$ & 1600 & .0 & $T-4$ & $04-15-84$ & 0800 & .0 \\
\hline$T-4$ & $03-26-84$ & 0800 & .0 & $T-4$ & $04-16-84$ & 0800 & .0 \\
\hline$T-4$ & $03-26-84$ & 1200 & .0 & $T-4$ & $04-20-84$ & 0800 & .0 \\
\hline $\mathrm{T}-4$ & $03-26-84$ & 1600 & .0 & $T-4$ & $04-21-84$ & $0800 *$ & .0 \\
\hline$T-4$ & $03-27-84$ & 0800 & .0 & $T-4$ & $04-23-84$ & 0800 & .0 \\
\hline$T-4$ & $03-27-84$ & 1600 & .0 & $T-4$ & $04-27-84$ & 0800 & .0 \\
\hline $\mathrm{T}-4$ & $03-28-84$ & 1200 & .0 & $\mathrm{~T}-4$ & $05-07-84$ & 0800 & .0 \\
\hline $\mathrm{T}-4$ & $03-29-84$ & 1600 & .0 & $T-4$ & $05-10-84$ & 0800 & .0 \\
\hline$T-4$ & $03-30-84$ & 0800 & .0 & $T-4$ & $05-11-84$ & 0800 & .0 \\
\hline$T-4$ & $03-30-84$ & 1600 & .0 & $\mathrm{~T}-4$ & $05-12-84$ & 0800 & .0 \\
\hline$T-5$ & $02-15-84$ & 0800 * & 0.0 & $\mathrm{~T}-5$ & $02-27-84$ & 0800 & 0.0 \\
\hline$T-5$ & $02-17-84$ & 0800 & .0 & $T-5$ & $02-29-84$ & 0800 & .0 \\
\hline$T-5$ & $02-19-84$ & 0800 & .0 & $T-5$ & $03-04-84$ & 0800 & .0 \\
\hline$T-5$ & $02-21-84$ & 0800 & .0 & $T-5$ & $03-06-84$ & 0800 & .0 \\
\hline$T-5$ & $02-23-84$ & 0800 & .0 & $T-5$ & $03-12-84$ & 0800 & .0 \\
\hline$T-5$ & $02-25-84$ & 0800 & .0 & $T-5$ & $03-24-84$ & 0800 & .0 \\
\hline
\end{tabular}


and the shape of the concentration hydrographs. Variable background fluorescence in the ground water caused some interference and made the measurement of RWT questionable below about 0.5 micrograms per liter. The RWT analyses were used to select samples to be analyzed for other tracers (table 4).

Table 4.--Chemical analyses of tracers

[Concentrations in milligrams per liter; a dash indicates no analysis made]

\begin{tabular}{|c|c|c|c|c|c|}
\hline $\begin{array}{l}\text { Well } \\
\text { No. }\end{array}$ & $\begin{array}{l}\text { Sample } \\
\text { date }\end{array}$ & $\begin{array}{l}\text { Trifluoro- } \\
\text { acetate }\end{array}$ & $\begin{array}{l}\text { Penta- } \\
\text { fluoro- } \\
\text { propionate }\end{array}$ & $\begin{array}{c}\text { m-Trifluoro- } \\
\text { methyl- } \\
\text { benzoate }\end{array}$ & Pyridone \\
\hline$T-1$ & Feb. 3,1984 & $<0.05$ & $<0.05$ & $<0.1$ & $<0.004$ \\
\hline$T-1$ & Feb. 7,1984 & 3.3 & 3.4 & 2.2 & .002 \\
\hline$T-1$ & Feb. 9,1984 & 5.2 & 4.9 & 4.0 & .010 \\
\hline$T-1$ & Feb. 11, 1984 & -- & -- & 5.6 & .024 \\
\hline$T-1$ & Feb. 13, 1984 & 8.6 & 8.8 & 5.6 & .040 \\
\hline$T-1$ & Feb. 15, 1984 & 8.6 & 8.5 & 6.4 & .064 \\
\hline$T-1$ & Feb. 17, 1984 & 7.9 & 8.3 & 6.4 & .070 \\
\hline$T-1$ & Feb. 19, 1984 & 6.8 & 7.0 & 7.4 & .068 \\
\hline$T-1$ & Feb. 21, 1983 & 7.3 & 7.4 & 5.4 & .068 \\
\hline$T-1$ & Mar. 6,1984 & -- & 5.9 & 4.0 & .052 \\
\hline$T-1$ & Mar. 11, 1984 & -- & -- & 4.2 & .044 \\
\hline$r-1$ & Mar. 21, 1984 & 2.0 & 2.2 & 1.6 & .019 \\
\hline
\end{tabular}

Rhodamine WT was first detected in well $\mathrm{T}-1$ on February 7 and in $\mathrm{T}-2$ on February 9, and the concentrations increased sharply until February 13 when both wells showed peaks on the concentration hydrographs (fig. 4). The RWT concentration in well $\mathrm{T}-1$ increased again after February 15 and was at a maximum on February 21.

The distance from the injection well (601) and the closest observation well $(T-1)$ is 87 feet. The distance between well 601 and well T-2 is 110 feet. The February 7 and February 9 dates for arrival of the leading edge of the dye cloud give a time of travel of 14 days to well $\mathrm{T}-1$ and 16 days to well T-2. The computed velocities are 6.2 and 6.9 feet per day, respectively.

\section{Factors Affecting Measured Velocity}

The differences in shapes of the RWT hydrographs and the large differences in concentrations for wells $T-1$ and $T-2$ (fig. 4) may be attributable to physical characteristics of the wells and the water bearing materials, and to the chemical characteristics of the dye. Dispersion and insensitive analytical procedures may account for chloride not being detected. 


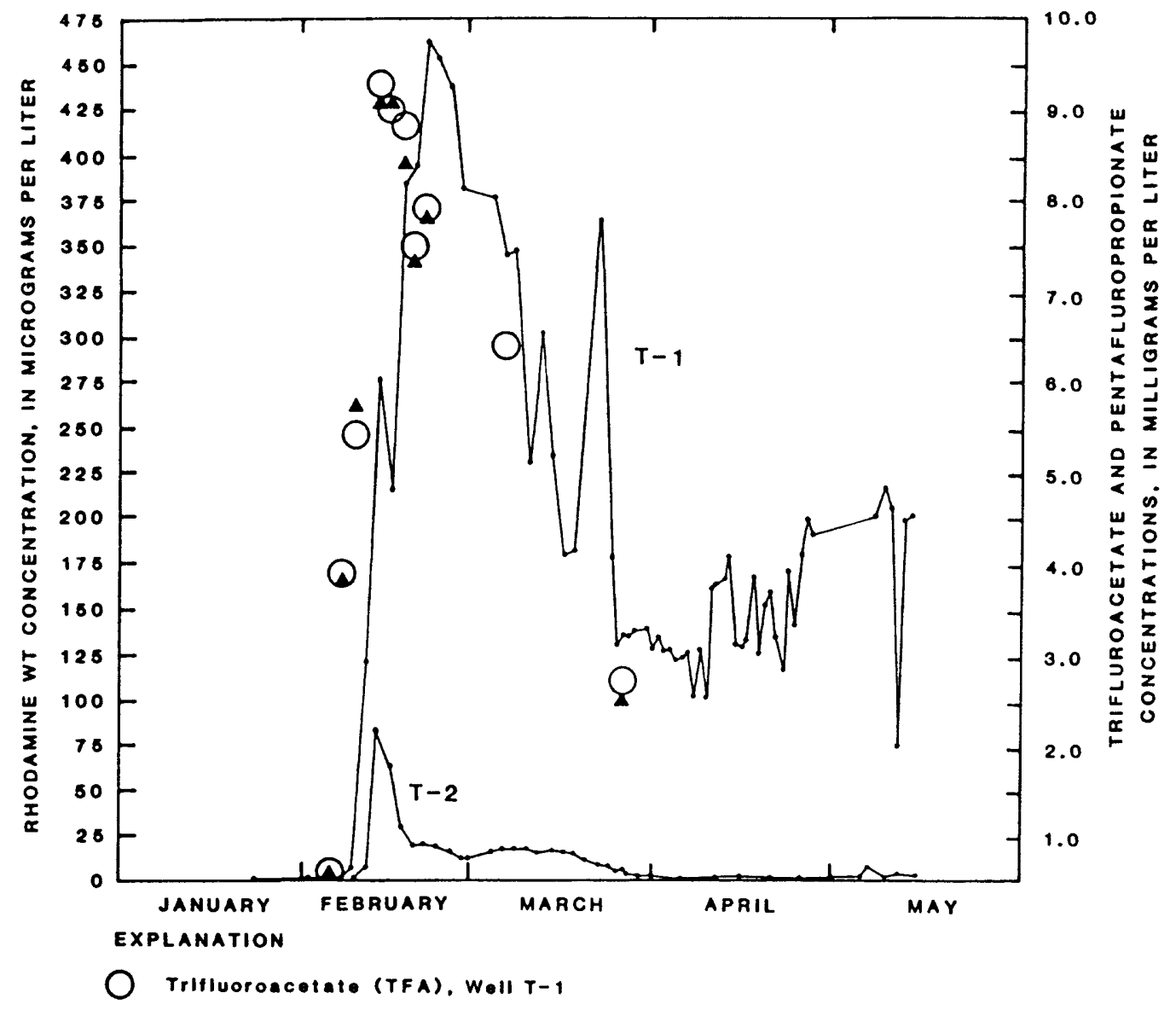

$\Delta$ Pentafluoropropionate (PFP), Well $T-1$

Rhodamine WT

Figure 4.--Concentration hydrographs of Rhodamine WT dye. 
The test kit used for analyses of chloride had a detection limit of about 35 milligrams per liter. Apparently dilution was sufficient to decrease the concentration at the observation wells below the detection limit. Once dye was visible at well $\mathrm{T}-1$, no further efforts were made to use chloride to estimate ground-water velocity.

Well T-1 may be closer than well $\mathrm{T}-2$ to the center of the flow path from well 601. The water levels (table 1) indicate a gradient along the line from well $\mathrm{T}-3$ to $\mathrm{T}-5$, however, no dye was detected in wells $\mathrm{T}-3, \mathrm{~T}-4$, or $\mathrm{T}-5$. This supports the concept of a very narrow preferential flow path eastward from well 601.

The RWT dye may have sorbed onto silts and clays in the pebbly-sand unit. The tracers TFA and PFP had beginning concentrations of about the same magnitude as RWT but their concentrations ( $\mathrm{fig}$. 4) in samples from well $\mathrm{T}-1$ are much higher than the dye concentrations. Adsorption of the dye onto silts and clays could cause much of the differences in concentrations between wells $T-1$ and $\mathrm{T}-2$.

The tracer solution had a higher specific gravity than the ground water and may not have been evenly dispersed vertically when it reached the observation wells. The 2-foot observation-well screens only screened part of the sand unit below the water table. Vertical stratification of the dye in the ground water could account for some of the variations in concentration at well T-1 and some of the differences in concentration between wells $\mathrm{T}-1$ and $\mathrm{T}-2$.

During injection, the tracers may have become separated into two overlapping dye clouds. About 2.5 gallons of tracer solution (including rinse water) could raise the water level about 4 feet in the 4-inch injection well. The pressure head caused by the higher water level would cause an equal

2.5-galion volume of the mixed column of solution to be displaced from the well into the sand, either above or below the water table, as the water level in the well returned to equilibrium. Water that moved through the geologic materials and flushed the tracers from the well may have displaced the remaining tracer solution at a different rate than the first 2.5 gallons.

Because the sampling frequency was once daily at 8:00 a.m. until February 15, the dye cloud could have arrived at well $\mathrm{T}-1$ anytime after 8:00 a.m. February 6 , and the two computed velocities might have been nearly equal. The maximum measured ground-water velocities are considered to be the best estimates for the purpose of predicting the traveltime of conservative contaminants. The velocity of the leading edge of the RWT dye cloud between wells 601 and $\mathrm{T}-2$ was 6.9 feet per day $(2,500 \mathrm{ft} / \mathrm{yr})$, assuming a linear flow path between wells. The velocity based on the arrival time of the peak concentration was about 5.8 feet per day $(2,100 \mathrm{ft} / \mathrm{yr})$, also assuming a linear flow path. The assumption of a linear flow path may not be fully justified, and the actual velocities along flow paths may be somewhat higher. 
Ground-water flow in unconsolidated glacial deposits at a low-level radioactive-waste disposal site near Sheffield, Illinois, is generally to the east-northeast. Most of the ground water moves through a pebbly-sand unit of the Toulon Member of the Glasford Formation. Measurements of ground-water flow velocity were needed to help define the hydrogeology and to provide information for site planners to make knowledgeable decisions with regard to potential radionuclide migration.

Observation wells were installed offsite to the northeast to inject tracers and collect samples for tracer analyses. Ground-water velocity through the pebbly-sand was computed from the time of travel of Rhodamine WT dye between wells 110 feet apart.

The leading edge of the Rhodamine WT dye cloud traveled the 110 feet through the pebbly sand in 16 days. This gives a computed ground-water velocity of 6.9 feet per day $(2,500$ feet per year) at the measurement site, assuming a linear flow path between the wells. Velocities along actual flow paths may be higher.

\section{REFERENCES CITED}

Foster, J. B., Erickson, J. R., and Healy, R. W., 1984, Hydrology of a lowlevel radioactive-waste disposal site near Sheffield, Illinois: U.S. Geological Survey Water-Resources Investigations Report 83-4125, 83 p.

Foster, J. B., Garklavs, George, and Mackey, G. W., 1984, Hydrogeologic setting east of a low-level radioactive-waste disposal site near Sheffield, Illinois: U.S. Geological survey water-Resources Investigations Report 84-4183, 20 p. 